\title{
Chiral-spin symmetry emergence in baryons and eigenmodes of the Dirac operator
}

\author{
M. Catillo, L. Ya. Glozman, and C. B. Lang \\ Institute of Physics, University of Graz, 8010 Graz, Austria
}

(Received 7 April 2019; published 29 May 2019)

\begin{abstract}
Truncating the low-lying modes of the lattice Dirac operator results in an emergence of the chiral-spin symmetry $S U(2)_{C S}$ and its flavor extension $S U\left(2 N_{F}\right)$ in hadrons. These are symmetries of the quarkchromoelectric interaction and include chiral symmetries as subgroups. Hence the quark-chromomagnetic interaction, which breaks both symmetries, is located at least predominantly in the near-zero modes. Using as a tool the expansion of propagators into eigenmodes of the Dirac operator we here analytically study effects of a gap in the eigenmode spectrum on baryon correlators. We find that both $U(1)_{A}$ and $S U(2)_{L} \times S U(2)_{R}$ emerge automatically if there is a gap around zero. Emergence of larger $S U(2)_{C S}$ and $S U(4)$ symmetries requires in addition a microscopical dynamical input about the higher-lying modes and their symmetry structure.
\end{abstract}

DOI: 10.1103/PhysRevD.99.094040

\section{INTRODUCTION}

In a number of lattice spectroscopical studies with a chirally invariant Dirac operator upon artificial truncation of the lowest modes of the Dirac operator [1,2] a large degeneracy was discovered in mesons [3-5] and baryons [6]. Corresponding symmetry groups, $S U(2)_{C S}$ and $S U\left(2 N_{F}\right)$ $[7,8]$, turned out to be larger than the chiral symmetry $S U\left(N_{F}\right)_{L} \times S U\left(N_{F}\right)_{R} \times U(1)_{A}$ of the QCD Lagrangian. The chiral-spin symmetry group $S U(2)_{C S}$ has $U(1)_{A}$ as a subgroup while its flavor extension $S U\left(2 N_{F}\right)$ contains both $S U\left(N_{F}\right)_{L} \times S U\left(N_{F}\right)_{R} \times U(1)_{A}$ and $S U(2)_{C S}$ as subgroups. The chiral-spin transformations from $S U(2)_{C S}$ includes rotations that mix the left- and right-handed components of the quark field. Obviously these symmetries are not symmetries of a free Dirac equation or of the QCD Lagrangian. However, they are symmetries of the Lorentzinvariant fermion charge operator and (in a given reference frame) of the quark-chromoelectric interaction while the interaction of quarks with the chromomagnetic field and the quark kinetic term break them. Consequently the emergence of $S U(2)_{C S}$ and $S U\left(2 N_{F}\right)$ upon truncation of the low-lying modes tells that while the confining quark-electric interaction is distributed among all modes of the Dirac operator, the quark-magnetic interaction is located at least predominantly in the near-zero modes. Some unknown microscopic dynamics should be responsible for this phenomenon.

Published by the American Physical Society under the terms of the Creative Commons Attribution 4.0 International license. Further distribution of this work must maintain attribution to the author(s) and the published article's title, journal citation, and DOI. Funded by SCOAP .
These symmetries emerge naturally, i.e., without any explicit truncation, in hot QCD above the pseudocritical temperature [9-11], where the near-zero modes of the Dirac operator are suppressed by temperature [12]. Consequently elementary objects in that range are not free quarks and gluons but rather chirally symmetric quarks bound by the chromoelectric field into color singlet objects, such as a "string."

According to the Banks-Casher relation [13] the chiral symmetry breaking quark condensate is proportional to the density of the near-zero modes. A gap in the low-lying Dirac eigenmode spectrum induces restoration of $S U\left(N_{F}\right)_{L} \times S U\left(N_{F}\right)_{R}$ symmetry. It was shown that it also induces restoration of $U(1)_{A}$ in the $J=0$ mesons [14]. Analytical study of the $J=0$ and $J=1$ isovector meson propagators in terms of the eigenmodes of the Dirac operator revealed that all meson correlators that are connected by the $U(1)_{A}$ and/or $S U(2)_{L} \times S U(2)_{R}$ transformations get necessarily degenerate if such a gap exists in the Dirac spectrum [15]. However, a possible emergence of $S U(2)_{C S}$ and of $S U\left(2 N_{F}\right)$ requires further dynamical properties encoded in certain matrix elements. Here we extend this analysis to baryons and show that the same conclusions remain valid in this case as well.

\section{CHIRAL-SPIN SYMMETRY}

The $S U(2)_{C S}$ chiral-spin transformations for quarks are given by

$$
\psi \rightarrow \psi^{\prime}=\exp \left(i \frac{\varepsilon^{n} \Sigma^{n}}{2}\right) \psi
$$


where generators, defined in the Dirac spinor space, are

$$
\boldsymbol{\Sigma}=\left\{\gamma_{\mathbf{k}},-\mathrm{i} \gamma_{\mathbf{5}} \gamma_{\mathbf{k}}, \gamma_{\mathbf{5}}\right\}
$$

Here $\gamma_{k}, k=1,2,3,4$, are hermitian Euclidean gamma matrices, obeying the anticommutation relations

$$
\gamma_{i} \gamma_{j}+\gamma_{j} \gamma_{i}=2 \delta_{i j} ; \quad \gamma_{5}=\gamma_{1} \gamma_{2} \gamma_{3} \gamma_{4}
$$

Different $k$ define four-dimensional representations that can be reduced into two-dimensional irreducible ones. The $\mathfrak{s} \mathfrak{u}(2)$ algebra

$$
\left[\Sigma^{a}, \Sigma^{b}\right]=2 \mathrm{i} \epsilon^{a b c} \Sigma^{c}
$$

is satisfied for any $k$ in Eq. (2).

$U(1)_{A}$ is a subgroup of $S U(2)_{C S}$. The $S U(2)_{C S}$ transformations mix the left- and right-handed fermions and different representations of the Lorentz group. The free massless quark Lagrangian and Dirac equation do not have this symmetry.

Extending the direct product $S U(2)_{C S} \times S U\left(N_{F}\right)$ one obtains an $S U\left(2 N_{F}\right)$ group. The chiral symmetry group of QCD $S U\left(N_{F}\right)_{L} \times S U\left(N_{F}\right)_{R} \times U(1)_{A}$ is a subgroup of $S U\left(2 N_{F}\right)$. The $S U\left(2 N_{F}\right)$ transformations are given by

$$
\psi \rightarrow \psi^{\prime}=\exp \left(\frac{\mathrm{i}}{2} \epsilon^{m} T^{m}\right) \psi
$$

where $m=1,2, \ldots,\left(2 N_{F}\right)^{2}-1$. The set of $\left(2 N_{F}\right)^{2}-1$ generators is

$$
T^{m}=\left\{\left(\tau^{a} \otimes \mathbb{1}_{D}\right),\left(\mathbb{1}_{F} \otimes \Sigma^{n}\right),\left(\tau^{a} \otimes \Sigma^{n}\right)\right\},
$$

with the flavor generators $\tau$ with flavor index $a$ and $n=1$, 2,3 is the $S U(2)_{C S}$ index.

The fundamental vector of $S U\left(2 N_{F}\right)$ at $N_{F}=2$ is

$$
\psi=\left(\begin{array}{c}
u_{\mathrm{R}} \\
u_{\mathrm{L}} \\
d_{\mathrm{R}} \\
d_{\mathrm{L}}
\end{array}\right) .
$$

The $S U(2)_{C S}$ and $S U\left(2 N_{F}\right)$ groups are not symmetries of the QCD Lagrangian as a whole.

In a given reference frame the quark-gluon interaction Lagrangian in Minkowski space can be split into temporal and spatial parts:

$$
\bar{\psi} \gamma^{\mu} D_{\mu} \psi=\bar{\psi} \gamma \gamma^{0} D_{0} \psi+\bar{\psi} \gamma^{i} D_{i} \psi
$$

Here $D_{\mu}$ is a covariant derivative that includes interaction of the quark field $\psi$ with the gluon field $\boldsymbol{A}_{\mu}$,

$$
D_{\mu} \psi=\left(\partial_{\mu}-i g \frac{\boldsymbol{t} \cdot \boldsymbol{A}_{\mu}}{2}\right) \psi .
$$

The temporal term includes an interaction of the color-octet charge density

$$
\bar{\psi}(x) \gamma^{0} \frac{\boldsymbol{t}}{2} \psi(x)=\psi(x)^{\dagger} \frac{\boldsymbol{t}}{2} \psi(x)
$$

with the electric part of the gluonic gauge field. It is invariant under any unitary transformation acting in the Dirac and/or flavor spaces. In particular, it is a singlet under $S U(2)_{C S}$ and $S U\left(2 N_{F}\right)$ groups. The spatial part consists of a quark kinetic term and interaction with the magnetic part of the gauge field. It breaks $S U(2)_{C S}$ and $S U\left(2 N_{F}\right)$. We conclude that interaction of electric and magnetic components of the gauge field with fermions can be distinguished by symmetry.

In order to discuss the notions "electric" and "magnetic" one needs to fix the reference frame. An invariant mass of the hadron is the rest frame energy. Consequently, to discuss physics of hadron mass generation it is natural to use the hadron rest frame.

In Refs. [3-6] meson and baryon masses have been extracted from the asymptotic slope of the rest frame $t$-direction Euclidean correlator

$$
C_{\Gamma}(t)=\sum_{x, y, z}\left\langle\mathcal{O}_{\Gamma}(x, y, z, t) \mathcal{O}_{\Gamma}(\mathbf{0}, 0)^{\dagger}\right\rangle,
$$

where $\mathcal{O}_{\Gamma}(x, y, z, t)$ is an operator that creates a quarkantiquark pair for mesons or three quarks for baryons with fixed quantum numbers. Truncation of the nearzero modes of the Dirac operator resulted in the emergence of the $S U(2)_{C S}$ and $S U\left(2 N_{F}\right)$ symmetries in hadrons.

This implies that a confining $S U(2)_{C S^{-}}$and $S U\left(2 N_{F}\right)^{-}$ symmetric quark-electric interaction is distributed among all modes of the Dirac operator. At the same time the quarkmagnetic interaction that breaks both symmetries is located only in the low-lying modes. Consequently truncating the low-lying modes results in the emergence of symmetries in the spectrum of hadrons.

\section{CHIRAL AND CHIRAL-SPIN TRANSFORMATIONS OF NUCLEON OPERATORS}

In Ref. [6] the emergence of the $S U(2)_{C S}$ symmetry in nucleons upon truncation of the lowest-lying modes of the Dirac operator was studied on the lattice. In particular, it was demonstrated that correlators along the time direction 
TABLE I. List of Dirac structures for the $N$ baryon fields with scalar or pseudoscalar diquarks, where $I$ is the isospin and $J^{P}$ indicates spin and parity. The $s_{2}^{(i)}$ come from the relation $\gamma_{4} \Gamma_{2}^{(i) \dagger} \gamma_{4}=s_{2}^{(i)} \Gamma_{2}^{(i)}$.

\begin{tabular}{lcccc}
\hline \hline$I, J^{P}$ & $\Gamma_{1}^{(i)}$ & $\Gamma_{2}^{(i)}$ & $s_{2}^{(i)}$ & $i$ \\
\hline$N^{(i)}\left(\frac{1}{2}, \frac{1 \pm}{2}\right)$ & 1 & $C \gamma_{5}$ & +1 & 1 \\
& $\gamma_{5}$ & $C$ & -1 & 2 \\
& $\mathrm{i} \rrbracket$ & $C \gamma_{5} \gamma_{4}$ & +1 & 3 \\
& $\mathrm{i} \gamma_{5}$ & $C \gamma_{4}$ & +1 & 4 \\
\hline \hline
\end{tabular}

calculated with different nucleon operators that are not connected by chiral $U(1)_{A}$ and/or $S U(2)_{L} \times S U(2)_{R}$ transformations but are connected by the chiral-spin transformation (1) and (2) with $k=4$ get degenerate. As discussed in the Introduction our main objective here is to analyze which conditions would be sufficient for the emergence of chiral and chiral-spin symmetries in nucleons upon the low-mode truncation (or suppression). To this end we first classify the nucleon operators with respect to chiral and chiral-spin transformations. Such a classification of nucleon operators (with spin zero diquark) for $U(1)_{A}$, $S U(2)_{L} \times S U(2)_{R}$ and $S U(2)_{C S}, k=4$ transformations is discussed below.

A complete set of nucleon operators $(J=1 / 2, I=1 / 2$, $P= \pm 1$ ) with spin-zero diquarks consists of four operators [16] of the following form:

$$
N_{ \pm}^{(i)}=\epsilon_{a b c} \mathcal{P}_{ \pm} \Gamma_{1}^{(i)} u_{a}\left\{d_{b}^{T} \Gamma_{2}^{(i)} u_{c}-u_{b}^{T} \Gamma_{2}^{(i)} d_{c}\right\}
$$

where $\mathcal{P}_{ \pm}=\frac{1}{2}\left(\mathbb{1} \pm \gamma_{4}\right)$ is the parity projector. The matrices $\Gamma_{1}^{(i)}$ and $\Gamma_{2}^{(i)}$ are given in Table I. In our case the diquark $\left\{d_{b}^{T} \Gamma_{2}^{(i)} u_{c}-u_{b}^{T} \Gamma_{2}^{(i)} d_{c}\right\}$ has spin 0 and isospin $I=0$.

It is known that only two local nucleon operators are linearly independent if one takes into account the requirements of Lorentz and Fierz invariance [17]. However, the chiral-spin symmetry is not a symmetry of the Dirac equation and the chiral-spin transformations mix different irreducible representations of the Lorentz group. Consequently if one discusses properties of operators under the chiral-spin transformations one needs a complete set of such operators with respect to $S U(2)_{C S}$. Since a single-quark field transforms under a two-dimensional irreducible representation (1) and (2) of $S U(2)_{C S}, \quad k=4$, a complete set of three-quark nucleon interpolators with respect to $S U(2)_{C S}$ should contain eight independent operators of positive and negative parity because $\mathbf{2} \otimes \mathbf{2} \otimes \mathbf{2}=\mathbf{2}_{1} \oplus \mathbf{2}_{2} \oplus \mathbf{4}$. Such operators with the $J=0$ diquark are listed in Table I.

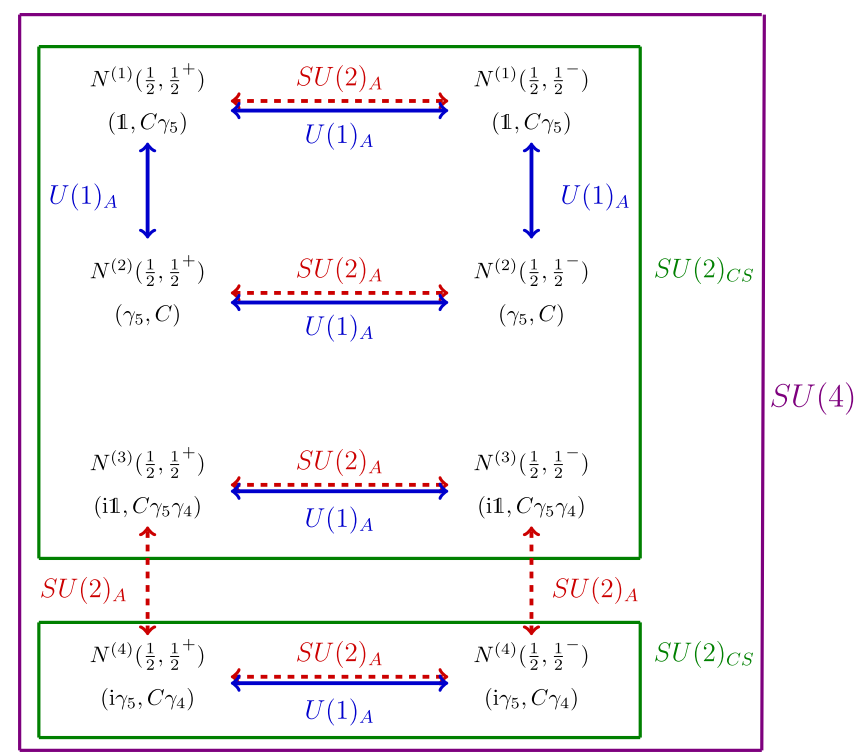

FIG. 1. The nucleons linked by dashed red arrows are connected by $S U(2)_{A}$, and those linked by blue arrows are connected by $U(1)_{A}$. The nucleons inside the green boxes are all connected via $S U(2)_{C S}$ and inside the violet box are connected via $S U(4)$.

Applying the $U(1)_{A}$ transformation on the given operator of Table I, one obtains a linear combination of some operators that are connected by blue arrows in Fig. 1. Consequently the operators connected by blue arrows form reducible representations of $U(1)_{A}$. The irreducible representations of $U(1)_{A}$ are one dimensional and can be obtained as certain linear combinations of operators connected by blue arrows.

The axial part of $S U(2)_{L} \times S U(2)_{R}$ [abbreviated as $\left.S U(2)_{A}\right]$ transforms the given operator into a linear superposition of operators connected by dashed red lines in Fig. 1. For example, both the operators of positive and negative parity $N^{(1)}\left(\frac{1}{2}^{2}, \frac{1}{2}^{ \pm}\right)$form a four-dimensional irreducible representation $(0,1 / 2)+(1 / 2,0)$ of the paritychiral group. The same is true for the operators $N^{(2)}\left(\frac{1}{2}, \frac{1}{2}\right)$.

For the operators $N^{(3)}\left(\frac{1}{2}, \frac{1}{2}^{ \pm}\right)$as well as $N^{(4)}\left(\frac{1}{2}, \frac{1}{2} \pm\right)$ the situation is a bit more complicated. Applying the $S U(2)_{L} \times S U(2)_{R}$ transformation on each of these operators, one obtains linear combinations of these operators as well as of $\Delta$ operators (isospin $I=3 / 2$ ) of the same spin. This is because certain linear combinations of $N^{(3)}\left(\frac{1}{2}, \frac{1}{2}\right)$ and $N^{(4)}\left(\frac{1}{2}, \frac{1}{2} \pm\right)$ form along with their $\Delta$ partners the irreducible representations $(1,1 / 2)+(1 / 2,1)$.

The $S U(2)_{C S}, k=4$ transformations connect all operators inside the green boxes of Fig. 1. Finally the $S U(4)$ transformations connect all eight operators of Fig. 1 along with the respective $\Delta$ partners.

Below we present a set of nucleon operators that transform under irreducible representations of $S U(2)_{C S}, k=4$ [16]. These operators are linear combinations of the operators from Table I: 


$$
\begin{aligned}
B_{2_{1}}(-1 / 2) & =\frac{1}{4 \sqrt{2}} \gamma_{-}\left[-\left(N_{+}^{(1)}-N_{-}^{(1)}\right)+\left(N_{+}^{(2)}-N_{-}^{(2)}\right)-\mathrm{i}\left(N_{+}^{(3)}+N_{-}^{(3)}\right)+\mathrm{i}\left(N_{+}^{(4)}+N_{-}^{(4)}\right)\right] \\
B_{2_{1}}(1 / 2) & =\frac{1}{4 \sqrt{2}} \gamma_{-}\left[\left(N_{+}^{(1)}+N_{-}^{(1)}\right)-\left(N_{+}^{(2)}+N_{-}^{(2)}\right)+\mathrm{i}\left(N_{+}^{(3)}-N_{-}^{(3)}\right)-\mathrm{i}\left(N_{+}^{(4)}-N_{-}^{(4)}\right)\right] \\
B_{2_{2}}(-1 / 2) & =\frac{1}{8} \sqrt{\frac{2}{3}} \gamma_{-}\left[-\left(N_{+}^{(1)}-N_{-}^{(1)}\right)+\left(N_{+}^{(2)}-N_{-}^{(2)}\right)-\mathrm{i}\left(N_{+}^{(3)}+N_{-}^{(3)}\right)-3 \mathrm{i}\left(N_{+}^{(4)}+N_{-}^{(4)}\right)\right] \\
B_{2_{2}}(1 / 2) & =\frac{1}{8} \sqrt{\frac{2}{3}} \gamma_{-}\left[\left(N_{+}^{(1)}+N_{-}^{(1)}\right)-\left(N_{+}^{(2)}+N_{-}^{(2)}\right)+\mathrm{i}\left(N_{+}^{(3)}-N_{-}^{(3)}\right)+3 \mathrm{i}\left(N_{+}^{(4)}-N_{-}^{(4)}\right)\right] \\
B_{4}(-3 / 2) & =\frac{1}{4} \gamma_{-}\left[\left(N_{+}^{(1)}+N_{-}^{(1)}\right)+\left(N_{+}^{(2)}+N_{-}^{(2)}\right)\right] \\
B_{4}(-1 / 2) & =\frac{1}{4} \sqrt{\frac{1}{3}} \gamma_{-}\left[\left(N_{+}^{(1)}-N_{-}^{(1)}\right)-\left(N_{+}^{(2)}-N_{-}^{(2)}\right)-2 \mathrm{i}\left(N_{+}^{(3)}+N_{-}^{(3)}\right)\right] \\
B_{4}(1 / 2) & =\frac{1}{4} \sqrt{\frac{1}{3} \gamma_{-}}\left[\left(N_{+}^{(1)}+N_{-}^{(1)}\right)-\left(N_{+}^{(2)}+N_{-}^{(2)}\right)-2 \mathrm{i}\left(N_{+}^{(3)}-N_{-}^{(3)}\right)\right] \\
B_{4}(3 / 2) & =\frac{1}{4} \gamma_{-}\left[\left(N_{+}^{(1)}-N_{-}^{(1)}\right)+\left(N_{+}^{(2)}-N_{-}^{(2)}\right)\right] .
\end{aligned}
$$

Explicitly these operators are

$$
\begin{aligned}
& B_{2_{1}}(-1 / 2)=\epsilon_{a b c} \sqrt{\frac{1}{2}} \gamma_{-}\left[\gamma_{4} u_{a}\left\{d_{b}^{T} C \gamma_{-} u_{c}\right\}+u_{a}\left\{d_{b}^{T} C \gamma_{4} \gamma_{-} u_{c}\right\}\right], \\
& B_{2_{1}}(1 / 2)=\epsilon_{a b c} \sqrt{\frac{1}{2}} \gamma_{-}\left[u_{a}\left\{d_{b}^{T} C \gamma_{+} u_{c}\right\}+\gamma_{4} u_{a}\left\{d_{b}^{T} C \gamma_{4} \gamma_{+} u_{c}\right\}\right], \\
& B_{2_{2}}(-1 / 2)=\epsilon_{a b c} \sqrt{\frac{1}{6}} \gamma_{-}\left[-2 u_{a}\left\{d_{b}^{T} C \gamma_{4} \gamma_{+} u_{c}\right\}-u_{a}\left\{d_{b}^{T} C \gamma_{4} \gamma_{-} u_{c}\right\}+\gamma_{4} u_{a}\left\{d_{b}^{T} C \gamma_{-} u_{c}\right\}\right] \text {, } \\
& B_{2_{2}}(1 / 2)=\epsilon_{a b c} \sqrt{\frac{1}{6}} \gamma_{-}\left[-2 \gamma_{4} u_{a}\left\{d_{b}^{T} C \gamma_{4} \gamma_{-} u_{c}\right\}-\gamma_{4} u_{a}\left\{d_{b}^{T} C \gamma_{4} \gamma_{+} u_{c}\right\}+u_{a}\left\{d_{b}^{T} C \gamma_{+} u_{c}\right\}\right] \text {, } \\
& B_{4}(-3 / 2)=-\epsilon_{a b c} \gamma_{-} u_{a}\left\{d_{b}^{T} C \gamma_{-} u_{c}\right\} \text {, } \\
& B_{4}(-1 / 2)=\epsilon_{a b c} \sqrt{\frac{1}{3}} \gamma_{-}\left[-u_{a}\left\{d_{b}^{T} C \gamma_{4} \gamma_{+} u_{c}\right\}+u_{a}\left\{d_{b}^{T} C \gamma_{4} \gamma_{-} u_{c}\right\}-\gamma_{4} u_{a}\left\{d_{b}^{T} C \gamma_{-} u_{c}\right\}\right] \text {, } \\
& B_{4}(1 / 2)=\epsilon_{a b c} \sqrt{\frac{1}{3} \gamma_{-}}\left[\gamma_{4} u_{a}\left\{d_{b}^{T} C \gamma_{4} \gamma_{-} u_{c}\right\}-\gamma_{4} u_{a}\left\{d_{b}^{T} C \gamma_{4} \gamma_{+} u_{c}\right\}+u_{a}\left\{d_{b}^{T} C \gamma_{+} u_{c}\right\}\right] \text {, } \\
& B_{4}(3 / 2)=\epsilon_{a b c} \gamma_{-} \gamma_{4} u_{a}\left\{d_{b}^{T} C \gamma_{+} u_{c}\right\} \text {. }
\end{aligned}
$$

Here $\gamma_{ \pm}=\frac{1}{2}\left(\mathbb{1} \pm \gamma_{5}\right)$ and $B_{r}\left(\chi_{z}\right)$ is the nucleon interpolator in the irreducible representation of dimension $r=$ $2 \chi+1$ of $S U(2)_{C S}$ and with chiral-spin index $\chi_{z}(z$ projection of the chiral-spin $\chi$ ). In (14) the curly brackets $\{\cdots\}$ mean antisymmetrization between $d_{b}$ and $u_{c}$ quarks as in (12). Upon the chiral-spin transformation (1) and (2) with $k=4$ only those nucleon operators are connected that belong to the same irreducible representation, as illustrated in Fig. 2.

\section{SPECTRAL DECOMPOSITION}

In this section we analyze the Euclidean nucleon propagators along $t$ direction upon truncation of the low-lying modes of the Dirac operator. We follow the procedure that was developed in Ref. [15] for a similar study of meson propagators. This approach is based on the spectral decomposition of the quark propagator in terms of the eigenmodes of the Dirac operator. The eigenmodes contain complete information about the interaction of a quark with a gluonic field.

We work in Euclidean spacetime and consider a hermitian massless Dirac operator $D_{0} \equiv \mathrm{i} \gamma_{\mu} D_{\mu}$. The eigenfunctions and eigenvalues of the Dirac operator are defined by the relation

$$
D_{0} \psi^{(n)}=\eta_{n} \psi^{(n)}
$$




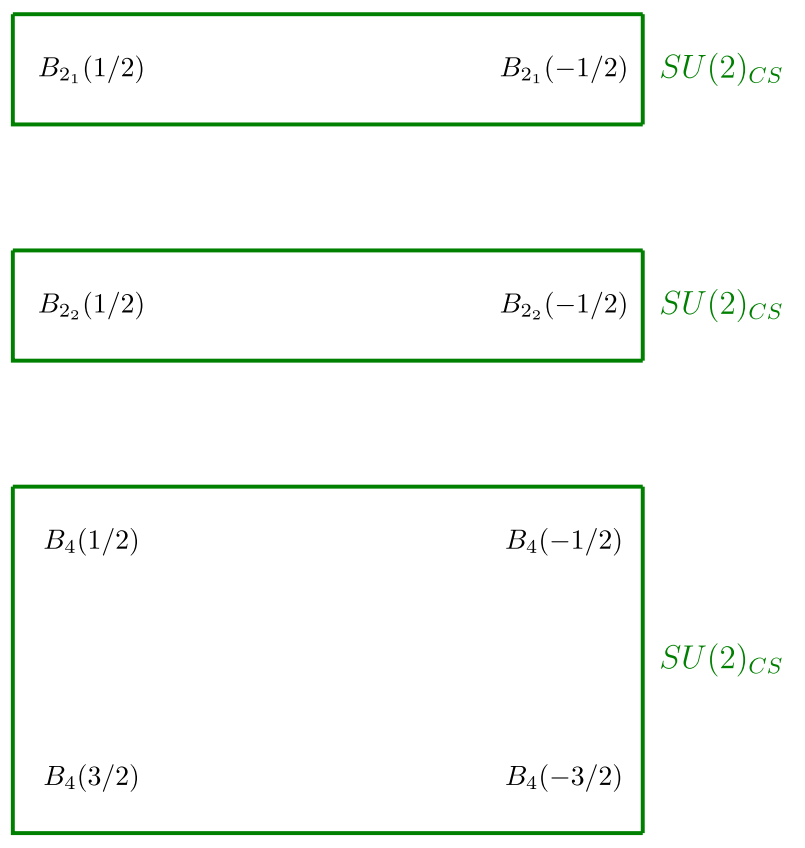

FIG. 2. Nucleons $B_{r}\left(\chi_{z}\right)$ in the irreducible representations of $S U(2)_{C S}$. Operators inside the green boxes form the basis of the corresponding irreducible representation and are connected via $S U(2)_{C S}$ transformations.

Because of $\left\{\gamma_{5}, D_{0}\right\}=0$, the eigenvalues come in pairs with opposite signs $\left(\eta_{n},-\eta_{n}\right)$ since

$$
D_{0} \gamma_{5} \psi^{(n)}=-\eta_{n} \gamma_{5} \psi^{(n)} \text {. }
$$

In the following we will use the notation $\eta_{-n} \equiv-\eta_{n}$. Here and in the rest of this work we assume that the Dirac operator $D_{0}$ does not have exact zero modes in its spectrum, which is equivalent to selecting gauge configurations with zero global topological charge. The contribution of exact zero modes to observables vanishes in the thermodynamic limit. Therefore in Eqs. (15) and (16), $\eta_{n} \neq 0$, for all $\psi^{(n)}$.

The full Dirac operator for a quark field with mass $m$ can be decomposed as

$$
\begin{aligned}
D & =D_{0}+\mathrm{i} m=\sum_{n}\left(\eta_{n}+\mathrm{i} m\right) \psi^{(n)} \psi^{(n) \dagger} \\
& =\sum_{n>0}\left[\left(\eta_{n}+\mathrm{i} m\right) \psi^{(n)} \psi^{(n) \dagger}+\left(-\eta_{n}+\mathrm{i} m\right) \gamma_{5} \psi^{(n)} \psi^{(n) \dagger} \gamma_{5}\right],
\end{aligned}
$$

where we used (15) and (16).

Now we consider baryon propagators and their decomposition using (17) for a theory with two mass degenerate quark flavors. A general baryon interpolator [see Eq. (14)] can be written as

$$
O(x)=\sum_{i} c_{i} O^{(i)}(x)
$$

for some choice of the coefficients $c_{i} \in \mathbb{C}$, in which

$$
O^{(i)}(x)=\epsilon_{a b c} \hat{\Gamma}_{1}^{(i)} u_{a}\left\{d_{b}^{T} \Gamma_{2}^{(i)} u_{c}-u_{b}^{T} \Gamma_{2}^{(i)} d_{c}\right\}
$$

where $\hat{\Gamma}_{1}^{(i)}$ is given by a linear combination of products of Dirac matrices, $\Gamma_{2}^{(i)}$ is a generic product of gamma matrices, and it satisfies the relation $\gamma_{4} \Gamma_{2}^{(i) \dagger} \gamma_{4}=s_{2}^{(i)} \Gamma_{2}^{(i)}$, where $s_{2}^{(i)}= \pm 1$.

The propagator associated with the operators $O^{(i)}(x)$ and $O^{(j)}(y)$, after the application of the Wick contractions, is given by

$$
\begin{aligned}
& C^{(i, j)}(x, y)=\left\langle O^{(i)}(x) \bar{O}^{(j)}(y)\right\rangle_{A} \\
& =s_{2}^{(i)} \epsilon_{a b c} \epsilon_{a^{\prime} b^{\prime} c^{\prime}}\left(\tilde{\Gamma}_{1}^{(i)}\right)_{\xi \alpha}\left(\Gamma_{2}^{(i)}\right)_{\beta \gamma}\left(\Gamma_{2}^{(j)}\right)_{\gamma^{\prime} \beta^{\prime}}\left(\gamma_{4} \tilde{\Gamma}_{1}^{(j) \dagger}\right)_{\alpha^{\prime} \xi} \\
& \times\left[D_{u_{x a \alpha \mid y a^{\prime} \alpha^{\prime}}^{-1}}^{-1} D_{d_{x b \beta \mid y b^{\prime} \beta^{\prime}}^{-1}}^{-1} D_{u_{x c \gamma \mid y c^{\prime} \gamma^{\prime}}^{-1}}\right. \\
& -D_{u_{x a \alpha \mid y c^{\prime} \gamma^{\prime}}^{-1}}^{-1} D_{d_{x b \beta \mid y b^{\prime} \beta^{\prime}}^{-1}}^{-1} D_{u_{x c \mid y a^{\prime} \alpha^{\prime}}^{-1}}^{-1} \text {. }
\end{aligned}
$$

Furthermore we have called, e.g., $D_{u_{x a \alpha \mid y a^{\prime} \alpha^{\prime}}^{-1}=}=$ $\left\langle u_{x a \alpha} \bar{u}_{y a^{\prime} \alpha^{\prime}}\right\rangle_{A}$ the quark propagator of the up quark between the spacetime points $x$ and $y$, with color indices $a$ and $a^{\prime}$, and Dirac indices $\alpha$ and $\alpha^{\prime}$. In the case of two degenerate quark masses, then $D^{-1} \equiv D_{u}^{-1}=D_{d}^{-1}$.

In the absence of zero modes in the Dirac spectrum, the quark propagator $D^{-1}$ can be expanded [see Ref. [15] and Eq. (17)] as

$D_{x a \delta \mid y a^{\prime} \alpha^{\prime}}^{-1}=\sum_{n>0} f_{n} \psi_{x a \alpha}^{(n)} \psi_{y a^{\prime} \alpha^{\prime}}^{(n) \dagger}+f_{-n}\left(\gamma_{5}\right)_{\alpha \xi} \psi_{x a \xi}^{(n)} \psi_{y a^{\prime} \xi^{\prime}}^{(n) \dagger}\left(\gamma_{5}\right)_{\xi^{\prime} \alpha^{\prime}}$,

where

$$
\begin{gathered}
f_{n}=\frac{1}{\eta_{n}+\mathrm{i} m}=h_{n}-\mathrm{i} g_{n}, \\
f_{-n}=\frac{1}{\eta_{-n}+\mathrm{i} m}=-h_{n}-\mathrm{i} g_{n},
\end{gathered}
$$

with

$$
\begin{aligned}
& h_{n} \equiv h\left(m, \eta_{n}\right)=\frac{\eta_{n}}{m^{2}+\eta_{n}^{2}}, \\
& g_{n} \equiv g\left(m, \eta_{n}\right)=\frac{m}{m^{2}+\eta_{n}^{2}}, \quad n>0 .
\end{aligned}
$$

Substituting Eq. (21) in the full propagator

$$
C(x, y)=\langle O(x) \bar{O}(y)\rangle_{A}=\sum_{i, j} c_{i} c_{j}^{*} C^{(i, j)}(x, y),
$$

we can express it in terms of $h(m, \eta)$ and $g(m, \eta)$, 

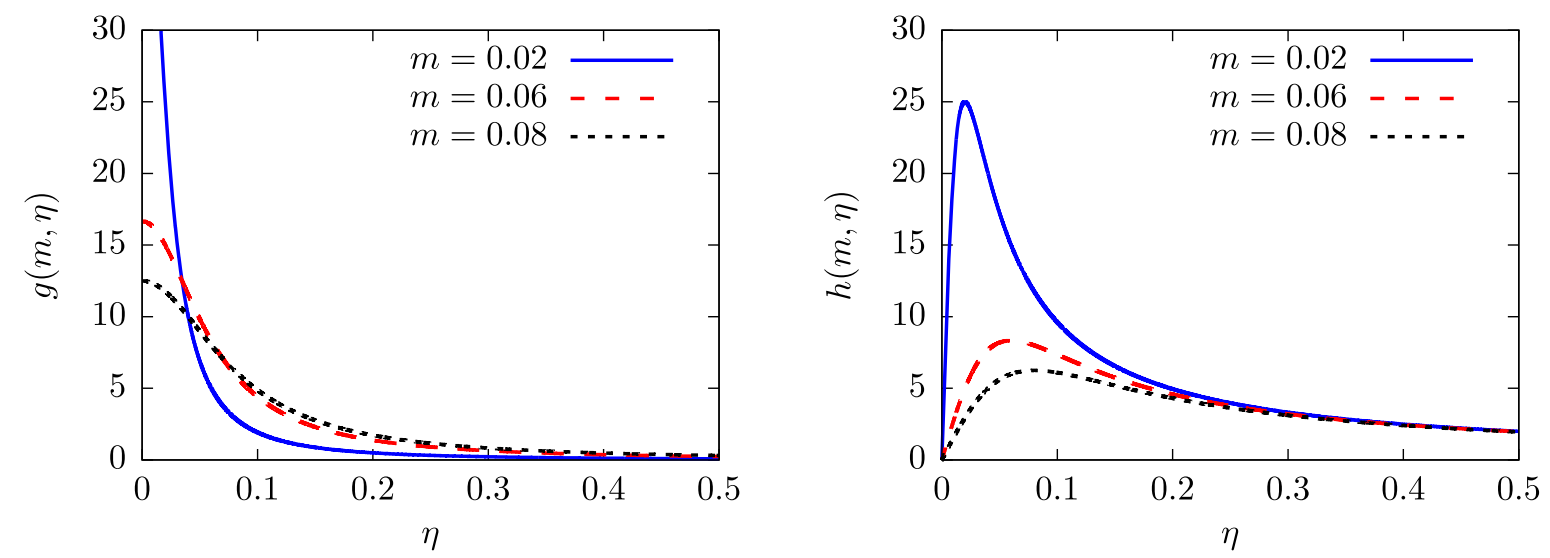

FIG. 3. $g(m, \eta)$ and $h(m, \eta)$ functions from Ref. [15] for $m=0.02$ (full line), 0.06 (dashed line), and 0.08 (dotted line).

$$
\begin{aligned}
C(x, y)= & \sum_{n>0, k>0, l>0}\left(g_{n} g_{k} g_{l} S^{g g g}(x, y)+g_{n} g_{k} h_{l} S^{g g h}(x, y)\right. \\
& \left.+g_{n} h_{k} h_{l} S^{g h h}(x, y)+h_{n} h_{k} h_{l} S^{h h h}(x, y)\right) .
\end{aligned}
$$

The functions $S^{g g g}(x, y), \quad S^{g g h}(x, y), \quad S^{g h h}(x, y), \quad$ and $S^{h h h}(x, y)$ contain the information about the eigenfunctions of the Dirac operator and the structure of the baryon field under consideration.

Therefore the correlator $C(x, y)$ has terms proportional to the $g(m, \eta)$ function, such as $g_{n} g_{k} g_{l} S^{g g g}(x, y)$, $g_{n} g_{k} h_{l} S^{g g h}(x, y)$, and $g_{n} h_{k} h_{l} S^{g h h}(x, y)$, that we call $g$ terms, and terms proportional only to the $h(m, \eta)$ function that we call $h$ terms. A sketch of these two functions for different mass values is shown in Fig. 3.

In the chiral limit $m \rightarrow 0$ the function $g(m, \eta)$ approaches the delta function $\frac{\pi}{2} \delta(\eta)$. Hence a gap around zero in the spectrum of the Dirac operator will induce the vanishing of the terms in Eq. (25) that contain at least one factor of $g$. In other words, all g terms in Eq. (25) vanish in the chiral limit upon truncation of the near-zero modes of the Dirac operator.

The $h(m, \eta)$ function is peaked at $\eta=m$ and falls slower compared to the $g(m, \eta)$ function at high eigenvalues $\eta$. Consequently while the $h(m, \eta)$ function still suppresses higher eigenvalues $\eta$, making a small hole in the Dirac eigenspectrum will not necessarily lead to the vanishing of the $h$ term in Eq. (25) in the chiral limit unless some additional suppressing dynamical factors are contained in $S^{h h h}(x, y)$.

In the following we call nucleon operators g equivalent if the difference of their propagators contains only $g$ terms.

\section{SPECTRAL DECOMPOSITION OF NUCLEON PROPAGATORS}

\section{A. Correlators of $\boldsymbol{N}^{(i)}$ operators}

Now we apply results of the previous section to correlators of nucleon operators from Table I. The details of the expansion in $g$ terms and $h$ terms of the nucleon propagators are given in Appendix A.

In Fig. 4 we show how the difference of two correlators (25) calculated with any two operators from Table I is expressed via the $g g g, g g h, g h h$, and $h h h$ terms. We see from Fig. 4 that all nucleons connected by $U(1)_{A}$ and/or $S U(2)_{A}$ transformations (see Fig. 1) are $g$ equivalent (for details see Appendixes B and C). Consequently a gap in the low-lying spectrum of the Dirac operator results (in the chiral limit) in the degeneracy of all correlators obtained with operators connected by dashed red and/or blue arrows in Fig. 1. We conclude that a gap in the Dirac spectrum implies necessarily restoration of both $U(1)_{A}$ and $S U(2)_{L} \times$ $S U(2)_{R}$ symmetries in nucleons. It is similar to the results for meson correlators obtained in [15]. Such degeneracies

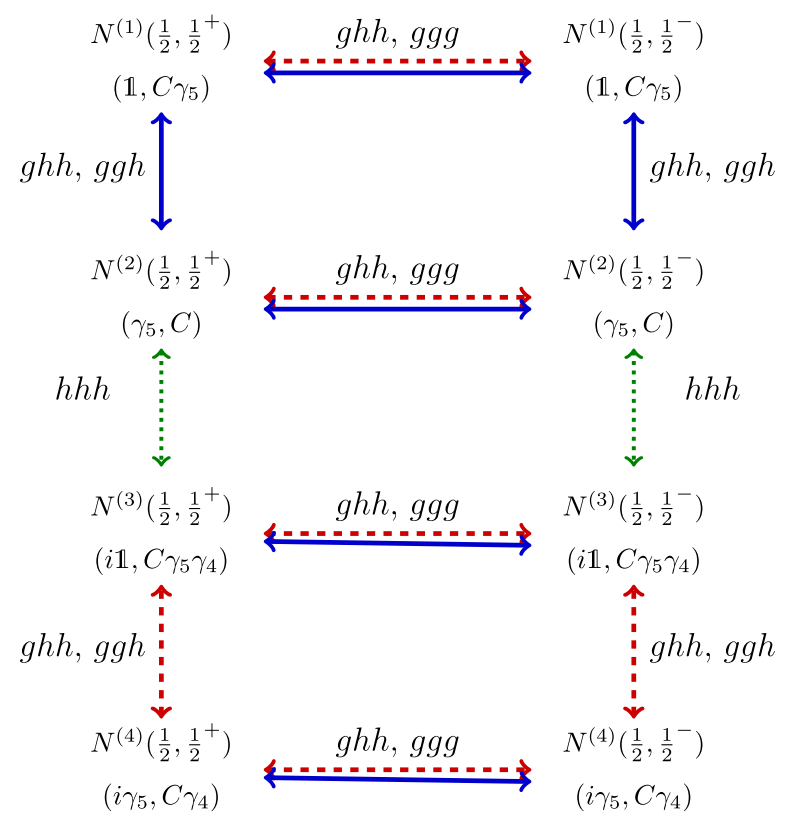

FIG. 4. $g$ and $h$ connections among nucleons of Table I. Below each nucleon we have indicated its $\Gamma$ structure, i.e., $\left(\Gamma_{1}^{(i)}, \Gamma_{2}^{(i)}\right)$. 
of the nucleon correlators have been observed on the lattice in Ref. [6].

Let us summarize. Restoration of $U(1)_{A}$ and $S U(2)_{L} \times$ $S U(2)_{R}$ symmetries in nucleon correlators (24) is necessarily provided by a gap in the spectrum of the Dirac operator, i.e., all $U(1)_{A}$ and $S U(2)_{L} \times S U(2)_{R}$ breaking dynamics are contained only in the near-zero modes.

However, the observations of Ref. [6] went essentially further than simply $U(1)_{A}$ and $S U(2)_{L} \times S U(2)_{R}$ restoration. It was noticed that larger symmetries $S U(2)_{C S}$ and $S U(4)$ emerge in baryon masses upon low-mode truncation.

From the analytical side we can now conclude the following. Comparing Fig. 4 with Fig. 1 we observe that, given a gap in the Dirac spectrum, the emergence of $S U(2)_{C S}$ and $S U(4)$ requires in addition that the $h$ term in the difference of two correlators connected by the $S U(2)_{C S}$ transformation (and not connected by the chiral transformations) should be at least strongly suppressed for higher-lying eigenmodes of the Dirac operator. While some suppression is indeed provided by the $h h h$ factor (see Fig. 3), this suppression is not as strong as in $g$ terms. In other words, a gap in the Dirac spectrum does not automatically imply emergence of the $S U(2)_{C S}$ and $S U(4)$ symmetries in correlators (25).

This result is not unexpected. In contrast to the chiral symmetries the $S U(2)_{C S}$ and $S U(4)$ symmetries are not covariant. They are symmetries of the quark-electric interaction in the given reference frame, while the quark kinetic term and the quark-magnetic interaction break them. They have been observed as symmetries of hadron masses upon low-mode truncation, i.e., symmetries of the rest frame correlation functions. The correlators (24) mix different reference frames in Minkowski space and only covariant symmetries, such as chiral symmetries, should persist in these correlators. Consequently to address the question of symmetries of hadron masses we need now to analyze the rest frame correlators (11). This means we need to study the correlators

$$
C_{ \pm}^{(i)}(t)=\sum_{x, y, z}\left\langle N_{ \pm}^{(i)}(x, y, z, t) \bar{N}_{ \pm}^{(i)}(\mathbf{0}, 0)\right\rangle
$$

where the sum $\sum_{x, y, z}$ is over all the space.

However, the summation over all spatial points $x, y, z$ does not convert an $h$ connection between the $N^{(2)}$ and $N^{(3)}$ operators in Fig. 4 into a $g$ connection. We do not get further $g$ equivalence as compared to the ones indicated in Fig. 4. The presence of a gap in the Dirac spectrum does not automatically imply the emergence of $S U(2)_{C S}$ and $S U(4)$.

In full QCD studies with the explicit removal of the lowlying modes in the propagators the $S U(2)_{C S}$ and $S U(4)$ symmetries were observed in the hadron spectrum [6]. This implies that a cancellation of $h h h$ terms occurs due to some additional $S U(2)_{C S}$ and $S U(4)$-symmetric microscopic dynamics, i.e., QCD dynamics beyond the chiral symmetry breaking dynamics dominated by the low modes. Such dynamics, as it follows from the symmetry classification of the QCD Lagrangian in Sec. II, should be related with the confining quark-chromoelectric interaction.

Let us summarize. Restoration of $U(1)_{A}$ and $S U(2)_{L} \times$ $S U(2)_{R}$ symmetries in nucleon correlators (24) is necessarily provided by a gap in the spectrum of the Dirac operator; i.e., all $U(1)_{A}$ and $S U(2)_{L} \times S U(2)_{R}$ breaking dynamics are contained only in the near-zero modes. The $S U(2)_{C S}$ and $S U(4)$ symmetries in the rest-frame correlators (26) do not automatically emerge. Their emergence requires some additional microscopical dynamical input that would guarantee that contributions of the high-lying modes are $S U(2)_{C S}$ and $S U(4)$ symmetric.

\section{B. $\boldsymbol{B}_{r}\left(\chi_{z}\right)$ baryon propagators}

In Fig. 2 we have reported irreducible $S U(2)_{C S}$ representations of the baryon operators defined in Eq. (14). Each of these operators is a $U(1)_{A}$ singlet, i.e., transforms into itself upon the $U(1)_{A}$ transformation. This is because by definition the $B_{r}\left(\chi_{z}\right)$ interpolators are eigenstates of $\gamma_{5}$ in the different representations $\boldsymbol{2}_{1}, \mathbf{2}_{2}$, and $\mathbf{4}$ of $S U(2)_{C S}$.

Regarding the $S U(2)_{L} \times S U(2)_{R}$, each operator from Fig. 2 is a linear combination of positive and negative parity operators (12). Different operators (12) belong to different irreducible representations of the parity-chiral group, as was discussed above, so no definite representation of $S U(2)_{L} \times S U(2)_{R}$ can be ascribed to the operators (14).

Now we apply a spectral decomposition of Sec. IV to the propagators built with the baryon operators (14)

$$
C(x, y)_{r, \chi_{z}}=\left\langle B_{r}\left(\chi_{z}\right)(x) \bar{B}_{r}\left(\chi_{z}\right)(y)\right\rangle .
$$

We find that the difference between two generic propagators $C(x, y)_{r, \chi_{z}}$ and $C(x, y)_{r^{\prime}, \chi_{z}^{\prime}}$, always contains $h h h$ terms. This means that a gap in the spectrum of the Dirac operator does not yet automatically imply emergence of the $S U(2)_{C S}$ and $S U(4)$ symmetries. This result is not unexpected since the correlators $C(x, y)_{r, \chi_{z}}$ mix different reference frames in Minkowski spacetime and only covariant symmetries can persist in such correlators. It is in complete agreement with the result obtained for the nucleon propagators; see Fig. 4.

Consequently we analyze now baryon correlators in the rest frame; i.e., we consider the correlators

$$
C(t)_{r, \chi_{z}}=\sum_{x, y, z}\left\langle B_{r}\left(\chi_{z}\right)(x, y, z, t) \bar{B}_{r}\left(\chi_{z}\right)(\mathbf{0}, 0)\right\rangle,
$$

where the sum $\sum_{x, y, z}$ is over all the space.

Under parity transformations the quark fields transform as 


$$
q_{x a \alpha} \stackrel{P}{\longrightarrow} q_{x a \alpha}^{P}=\left(\gamma_{4}\right)_{\alpha \beta} q_{\mathcal{P} x a \beta},
$$

where $\mathcal{P} x \equiv \mathcal{P}_{\mu \nu} x_{\nu}$ and $\mathcal{P}_{\mu \nu}=\operatorname{diag}(-1,-1,-1,1)$ is the parity operator; hence, if $x_{\mu}=(x, y, z, t)$, then $(\mathcal{P} x)_{\mu}=$ $(-x,-y,-z, t) \cdot q$ is a generic quark field. Applying the parity transformations (29) to the baryon operators in (14) we get the following relation for generic representation $r$ and chiral spin projection $\chi_{z}$ :

$$
B_{r}\left(\chi_{z}\right)(\mathcal{P} x)=\eta B_{r}^{P}\left(-\chi_{z}\right)(x)
$$

where we indicate $B_{r}\left(\chi_{z}\right)(\mathcal{P} x)=B_{r}\left(\chi_{z}\right)(-x,-y,-z, t)$ and $B_{r}^{P}\left(\chi_{z}\right)$ is the baryon operator $B_{r}\left(\chi_{z}\right)$ in (14), and we have substituted $u \rightarrow u^{P}$ and $d \rightarrow d^{P}$; see (29). In Eq. (30) $\eta= \pm 1$, depending on $r$ and $\chi_{z}$, and we used that $\gamma_{4} C=$ $-C \gamma_{4}$ and that $\gamma_{4} \gamma_{ \pm} \gamma_{4}=\gamma_{\mp}$. Plugging Eq. (30) in (28) we get

$$
\begin{aligned}
C(t)_{r, \chi_{z}} & =\sum_{x, y, z}\left\langle B_{r}\left(\chi_{z}\right)(x, y, z, t) \bar{B}_{r}\left(\chi_{z}\right)(\mathbf{0}, 0)\right\rangle \\
& =\sum_{x, y, z}\left\langle B_{r}\left(\chi_{z}\right)(-x,-y,-z, t) \bar{B}_{r}\left(\chi_{z}\right)(\mathbf{0}, 0)\right\rangle \\
& =\sum_{x, y, z}\left\langle B_{r}^{P}\left(-\chi_{z}\right)(x, y, z, t) \bar{B}_{r}^{P}\left(-\chi_{z}\right)(\mathbf{0}, 0)\right\rangle \\
& =\sum_{x, y, z}\left\langle B_{r}\left(-\chi_{z}\right)(x, y, z, t) \bar{B}_{r}\left(-\chi_{z}\right)(\mathbf{0}, 0)\right\rangle \\
& =C(t)_{r,-\chi_{z}}
\end{aligned}
$$

where in the third line we used Eq. (30). Since we are averaging over all possible quark fields we can remove the label $P$ in the last line of Eq. (31) (because parity is a symmetry of the QCD action and the measure in the average $\langle\cdot\rangle$ is parity invariant).

Equation (31) tells us that for a given irreducible representation $r$ of $S U(2)_{C S}$ we have $C(t)_{r, \chi_{z}}-C(t)_{r,-\chi_{z}}=0$, for all $\chi_{z}$. Hence in the rest frame the correlators for the baryons within the doublet $\mathbf{2}_{1}$ and $\mathbf{2}_{2}$ representations are equal. This is a general statement, irrespective of whether there is or there is not a gap in the spectrum of the Dirac operator. This fact does not mean, however, that the $S U(2)_{C S}$ symmetry is manifest in the rest-frame correlators, because in the representation 4 the correlators with $\chi_{z}= \pm 1 / 2$ are not equal to the correlators with $\chi_{z}= \pm 3 / 2$.
The presence of a gap in the Dirac spectrum does not automatically make the correlators with $\chi_{z}= \pm 1 / 2$ and with $\chi_{z}= \pm 3 / 2$ g equivalent. The emergence of $S U(2)_{C S}$ requires some additional suppression of matrix elements with higher-lying modes as was discussed in the previous subsection.

\section{CONCLUSIONS}

In this paper we have analyzed analytically, by expansion of the propagators into eigenmodes of the Dirac operator, which symmetries emerge in baryon correlators (masses) if there is a gap around zero in the spectrum of the Dirac operator. We have found that such a gap results necessarily in the emergence of chiral $U(1)_{A}$ and $S U(2)_{L} \times S U(2)_{R}$ symmetries in baryons.

Some specific dynamics in QCD leads to the accumulation of the near-zero modes, i.e., to the breaking of chiral symmetries. Given the $\gamma^{5}$ anticommutativity of the Euclidean Dirac operator we prove here that a gap in the Dirac eigenmode spectrum implies necessarily restoration of both $U(1)_{A}$ and $S U(2)_{L} \times S U(2)_{R}$ symmetries. The root of this statement is precisely the same as of the BanksCasher relation. We do not need to know which dynamics and why it leads to the accumulation of the nearzero modes.

Emergence of larger $S U(2)_{C S}$ and $S U(4)$ symmetries, which was observed on the lattice upon truncation of the near-zero modes of the Dirac operator and also at high temperatures without any truncation, requires that the electric interaction should be the most important for higher-lying modes. This is buried in the eigenfunctions of the Dirac operator and cannot be specified within the present approach which does not use any dynamical input.

\section{ACKNOWLEDGMENTS}

Support from the Austrian Science Fund (FWF) through Grants No. DK W1203-N16 and No. P26627-N27 is acknowledged.

\section{APPENDIX A: NUCLEON PROPAGATOR EXPANSION}

Using Eq. (20) and the expansion of the quark propagator in (21), we can get the expansion of the propagator for the nucleon interpolators given in Eq. (12) and specified in Table I. It is given by

$$
C\left(N_{ \pm}^{(i)}\right)=s_{2}^{(i)} \epsilon_{a b c} \epsilon_{a^{\prime} b^{\prime} c^{\prime}}\left(\gamma_{4} \Gamma_{1}^{(i) \dagger}\right)_{\alpha^{\prime} \omega}\left(\mathcal{P}_{ \pm}\right)_{\omega \epsilon}\left(\Gamma_{1}^{(i)}\right)_{\epsilon \alpha}\left(\Gamma_{2}^{(i)}\right)_{\beta \gamma}\left(\Gamma_{2}^{(i)}\right)_{\gamma^{\prime} \beta^{\prime}}\left[D_{x a \alpha \mid y a^{\prime} \alpha^{\prime}}^{-1} D_{x b \beta \mid y b^{\prime} \beta^{\prime}}^{-1} D_{x c \gamma \mid y c^{\prime} \gamma^{\prime}}^{-1}-D_{x a \alpha \mid y c^{\prime} \gamma^{\prime}}^{-1} D_{x b \beta \mid y b^{\prime} \beta^{\prime}}^{-1} D_{x c \gamma \mid y a^{\prime} \alpha^{\prime}}^{-1}\right]
$$


The last line of Eq. (A1) can be written as the following sum:

$$
\begin{aligned}
& D_{x a \alpha \mid y a^{\prime} \alpha^{\prime}}^{-1} D_{x b \beta \mid y b^{\prime} \beta^{\prime}}^{-1} D_{x c y \mid y c^{\prime} \gamma^{\prime}}^{-1}-D_{x a \alpha \mid y c^{\prime} \gamma^{\prime}}^{-1} D_{x b \beta \mid y b^{\prime} \beta^{\prime}}^{-1} D_{x c \gamma \mid y a^{\prime} \alpha^{\prime}}^{-1} \\
& =\sum_{n>0, k>0, l>0}\left[f_{n} f_{k} f_{l}\left[\psi_{x a \alpha}^{(n)} \psi_{y a^{\prime} \alpha^{\prime}}^{(n) \dagger} \psi_{x b \beta}^{(k)} \psi_{y b^{\prime} \beta^{\prime}}^{(k)} \psi_{x c \gamma}^{(l)} \psi_{y c^{\prime} \gamma^{\prime}}^{(l) \dagger}\right]+f_{n} f_{k} f_{-l}\left[\psi_{x a \alpha}^{(n)} \psi_{y a^{\prime} \alpha^{\prime}}^{(n) \dagger} \psi_{x b \beta}^{(k)} \psi_{y b^{\prime} \beta^{\prime}}^{(k) \dagger}\left(\gamma_{5}\right)_{\gamma \theta} \psi_{x c \theta}^{(l)} \psi_{y c^{\prime} \theta^{\prime}}^{(l) \dagger}\left(\gamma_{5}\right)_{\theta^{\prime} \gamma^{\prime}}\right]\right. \\
& +f_{n} f_{-k} f_{l}\left[\psi_{x a \alpha}^{(n)} \psi_{y a^{\prime} \alpha^{\prime}}^{(n) \dagger}\left(\gamma_{5}\right)_{\beta \omega} \psi_{x b \omega}^{(k)} \psi_{y b^{\prime} \omega^{\prime}}^{(k) \dagger}\left(\gamma_{5}\right)_{\omega^{\prime} \beta^{\prime}} \psi_{x c \gamma}^{(l)} \psi_{y c^{\prime} \gamma^{\prime}}^{(l) \dagger}\right]+f_{-n} f_{k} f_{l}\left[\left(\gamma_{5}\right)_{\alpha \xi} \psi_{x a \xi}^{(n)} \psi_{y a^{\prime} \xi^{\prime}}^{(n) \dagger}\left(\gamma_{5}\right)_{\xi^{\prime} \alpha^{\prime}} \psi_{x b \beta}^{(k)} \psi_{y b^{\prime} \beta^{\prime}}^{(k) \dagger} \psi_{x c \gamma}^{(l)} \psi_{y c^{\prime} \gamma^{\prime}}^{(l) \dagger}\right] \\
& +f_{n} f_{-k} f_{-l}\left[\psi_{x a \alpha}^{(n)} \psi_{y a^{\prime} \alpha^{\prime}}^{(n) \dagger}\left(\gamma_{5}\right)_{\beta \omega} \psi_{x b \omega}^{(k)} \psi_{y b^{\prime} \omega^{\prime}}^{(k) \dagger}\left(\gamma_{5}\right)_{\omega^{\prime} \beta^{\prime}}\left(\gamma_{5}\right)_{\gamma \theta} \psi_{x c \theta}^{(l)} \psi_{y c^{\prime} \theta^{\prime}}^{(l) \dagger}\left(\gamma_{5}\right)_{\theta^{\prime} \gamma^{\prime}}\right] \\
& +f_{-n} f_{k} f_{-l}\left[\left(\gamma_{5}\right)_{\alpha \xi} \psi_{x a \xi}^{(n)} \psi_{y a^{\prime} \xi^{\prime}}^{(n) \dagger}\left(\gamma_{5}\right)_{\xi^{\prime} \alpha^{\prime}} \psi_{x b \beta}^{(k)} \psi_{y b^{\prime} \beta^{\prime}}^{(k) \dagger}\left(\gamma_{5}\right)_{\gamma \theta} \psi_{x c \theta}^{(l)} \psi_{y c^{\prime} \theta^{\prime}}^{(l) \dagger}\left(\gamma_{5}\right)_{\theta^{\prime} \gamma^{\prime}}\right] \\
& +f_{-n} f_{-k} f_{l}\left[\left(\gamma_{5}\right)_{\alpha \xi} \psi_{x a \xi}^{(n)} \psi_{y a^{\prime} \xi^{\prime}}^{(n) \dagger}\left(\gamma_{5}\right)_{\xi^{\prime} \alpha^{\prime}}\left(\gamma_{5}\right)_{\beta \omega} \psi_{x b \omega}^{(k)} \psi_{y b^{\prime} \omega^{\prime}}^{(k) \dagger}\left(\gamma_{5}\right)_{\omega^{\prime} \beta^{\prime}} \psi_{x c \gamma}^{(l)} \psi_{y c^{\prime} \gamma^{\prime}}^{(l) \dagger}\right] \\
& +f_{-n} f_{-k} f_{-l}\left[\left(\gamma_{5}\right)_{\alpha \xi} \psi_{x a \xi}^{(n)} \psi_{y a^{\prime} \xi^{\prime}}^{(n) \dagger}\left(\gamma_{5}\right)_{\xi^{\prime} \alpha^{\prime}}\left(\gamma_{5}\right)_{\beta \omega} \psi_{x b \omega}^{(k)} \psi_{y b^{\prime} \omega^{\prime}}^{(k) \dagger}\left(\gamma_{5}\right)_{\omega^{\prime} \beta^{\prime}}\left(\gamma_{5}\right)_{\gamma \theta} \psi_{x c \theta}^{(l)} \psi_{y c^{\prime} \theta^{\prime}}^{(l) \dagger}\left(\gamma_{5}\right)_{\theta^{\prime} \gamma^{\prime}}\right] \\
& \text { - (same terms as above with } \left.\alpha^{\prime} \leftrightarrow \gamma^{\prime} \text { and } a^{\prime} \leftrightarrow c^{\prime}\right) \text { ]. }
\end{aligned}
$$

Using (22) we can rewrite the coefficients in front of the eigenfunction products in (A2) in terms of $g_{n}$ and $h_{n}$, i.e.,

$$
f_{n} f_{k} f_{l}=\mathrm{i} g_{n} g_{k} g_{l}-h_{n} g_{k} g_{l}-g_{n} h_{k} g_{l}-\mathrm{i} h_{n} h_{k} g_{h}-g_{n} g_{k} h_{l}-\mathrm{i} h_{n} g_{k} h_{l}-\mathrm{i} g_{n} h_{k} h_{l}+h_{n} h_{k} h_{l} ;
$$

moreover other coefficients can be found exploiting that $f_{-n}=-f_{n}^{*}$ [see Eq. (22)]. Therefore by linearity of (A2), we can get the expression of $C\left(N_{ \pm}^{(i)}\right)$ in terms proportional to $g_{n} g_{k} g_{l}, g_{n} g_{k} h_{l}, g_{n} h_{k} h_{l}$, and $h_{n} h_{k} h_{l}$.

\section{APPENDIX B: $C\left(N_{+}^{(i)}\right)-C\left(N_{-}^{(i)}\right)$}

The difference $C\left(N_{+}^{(i)}\right)-C\left(N_{-}^{(i)}\right)$ can be written using (A1) as

$$
\begin{aligned}
C\left(N_{+}^{(i)}\right)-C\left(N_{-}^{(i)}\right)= & s_{2}^{(i)} \epsilon_{a b c} \epsilon_{a^{\prime} b^{\prime} c^{\prime}}\left(\gamma_{4} \Gamma_{1}^{(i) \dagger} \gamma_{4}\right)_{\alpha^{\prime} \omega}\left(\left(\mathcal{P}_{+}\right)_{\omega \epsilon}+\left(\mathcal{P}_{-}\right)_{\omega \epsilon}\left(\Gamma_{1}^{(i)}\right)_{\epsilon \alpha}\left(\Gamma_{2}^{(i)}\right)_{\beta \gamma}\left(\Gamma_{2}^{(i)}\right)_{\gamma^{\prime} \beta^{\prime}}\right. \\
& \times\left[D_{x a \alpha \mid y a^{\prime} \alpha^{\prime}}^{-1} D_{x b \beta \mid y b^{\prime} \beta^{\prime}}^{-1} D_{x c \alpha^{\prime} \mid y c^{\prime} \gamma^{\prime}}^{-1}-D_{x a \alpha \mid y c^{\prime} \gamma^{\prime}}^{-1} D_{x b \beta \mid y b^{\prime} \beta^{\prime}}^{-1} D_{x c \alpha^{\prime} \mid y a^{\prime} \alpha^{\prime}}^{-1}\right] \\
= & (-1)^{i+1} s_{2}^{(i)} \epsilon_{a b c} \epsilon_{a^{\prime} b^{\prime} c^{\prime}} \delta_{\alpha^{\prime} \alpha}\left(\Gamma_{2}^{(i)}\right)_{\beta \gamma}\left(\Gamma_{2}^{(i)}\right)_{\gamma^{\prime} \beta^{\prime}}\left[D_{x a \alpha \mid y a^{\prime} \alpha^{\prime}}^{-1} D_{x b \beta \mid y b^{\prime} \beta^{\prime}}^{-1} D_{x c \alpha^{\prime} \mid y c^{\prime} \gamma^{\prime}}^{-1}-D_{x a \alpha \mid y c^{\prime} \gamma^{\prime}}^{-1} D_{x b \beta \mid y b^{\prime} \beta^{\prime}}^{-1} D_{x c \alpha^{\prime} \mid y a^{\prime} \alpha^{\prime}}^{-1}\right]
\end{aligned}
$$

where we used that $\gamma_{4} \mathcal{P}_{ \pm}= \pm \mathcal{P}_{ \pm},\left(\mathcal{P}_{+}\right)_{\omega \epsilon}+\left(\mathcal{P}_{-}\right)_{\omega \epsilon}=\delta_{\omega \epsilon}$ and that $\Gamma_{1}^{(i) \dagger} \Gamma_{1}^{(i)}=\mathbb{1}$, for all values of $i$; see Table I.

We expand the quark propagator according to (A2) and use $\psi^{(-n)}=\gamma_{5} \psi^{(n)}$ and $\gamma_{5} \Gamma_{2}^{(i)} \gamma_{5}=s_{5(i)} \Gamma_{2}^{(i)}$ with $s_{5(i)}^{2}=1$ to get

$$
\begin{aligned}
& C\left(N_{+}^{(i)}\right)-C\left(N_{-}^{(i)}\right)=(-1)^{i+1} s_{2}^{(i)} \epsilon_{a b c} \epsilon_{a^{\prime} b^{\prime} c^{\prime}} \delta_{\alpha^{\prime} \alpha}\left(\Gamma_{2}^{(i)}\right)_{\beta \gamma}\left(\Gamma_{2}^{(i)}\right)_{\gamma^{\prime} \beta^{\prime}} \\
& \times \sum_{n>0, k>0, l>0}\left[\left(f_{n} f_{k} f_{l}+f_{-n} f_{-k} f_{-l}\right)\left[\psi_{x a \alpha}^{(n)} \psi_{y a^{\prime} \alpha^{\prime}}^{(n) \dagger} \psi_{x b \beta}^{(k)} \psi_{y b^{\prime} \beta^{\prime}}^{(k) \dagger} \psi_{x c \gamma}^{(l)} \psi_{y c^{\prime} \gamma^{\prime}}^{(l) \dagger}-\psi_{x a \alpha}^{(n)} \psi_{y c^{\prime} \gamma^{\prime}}^{(n) \dagger} \psi_{x b \beta}^{(k)} \psi_{y b^{\prime} \beta^{\prime}}^{(k) \dagger} \psi_{x c \gamma}^{(l)} \psi_{y a^{\prime} \alpha^{\prime}}^{(l) \dagger}\right]\right. \\
& +\left(f_{n} f_{k} f_{-l}+f_{-n} f_{-k} f_{l}\right)\left[\psi_{x a \alpha}^{(n)} \psi_{y a^{\prime} \alpha^{\prime}}^{(n) \dagger} \psi_{x b \beta}^{(k)} \psi_{y b^{\prime} \beta^{\prime}}^{(k) \dagger}\left(\gamma_{5}\right)_{\gamma \theta} \psi_{x c \theta}^{(l)} \psi_{y c^{\prime} \theta^{\prime}}^{(l) \dagger}\left(\gamma_{5}\right)_{\theta^{\prime} \gamma^{\prime}}\right. \\
& \left.-\psi_{x a \alpha}^{(n)} \psi_{y c^{\prime} \gamma^{\prime}}^{(n) \dagger} \psi_{x b \beta}^{(k)} \psi_{y b^{\prime} \beta^{\prime}}^{(k) \dagger}\left(\gamma_{5}\right)_{\gamma \theta} \psi_{x c \theta}^{(l)} \psi_{y a^{\prime} \theta^{\prime}}^{(l) \dagger}\left(\gamma_{5}\right)_{\theta^{\prime} \alpha^{\prime}}\right] \\
& +\left(f_{n} f_{-k} f_{l}+f_{-n} f_{k} f_{-l}\right)\left[\psi_{x a \alpha}^{(n)} \psi_{y a^{\prime} \alpha^{\prime}}^{(n) \dagger}\left(\gamma_{5}\right)_{\beta \omega} \psi_{x b \omega}^{(k)} \psi_{y b^{\prime} \omega^{\prime}}^{(k) \dagger}\left(\gamma_{5}\right)_{\omega^{\prime} \beta^{\prime}} \psi_{x c \gamma}^{(l)} \psi_{y c^{\prime} \gamma^{\prime}}^{(l) \dagger}\right. \\
& \left.-\psi_{x a \alpha}^{(n)} \psi_{y c^{\prime} \gamma^{\prime}}^{(n) \dagger}\left(\gamma_{5}\right)_{\beta \omega} \psi_{x b \omega}^{(k)} \psi_{y b^{\prime} \omega^{\prime}}^{(k) \dagger}\left(\gamma_{5}\right)_{\omega^{\prime} \beta^{\prime}} \psi_{x c \gamma}^{(l)} \psi_{y a^{\prime} \alpha^{\prime}}^{(l) \dagger}\right] \\
& +\left(f_{-n} f_{k} f_{l}+f_{n} f_{-k} f_{-l}\right)\left[\left(\gamma_{5}\right)_{\alpha \xi} \psi_{x a \xi}^{(n)} \psi_{y a^{\prime} \xi^{\prime}}^{(n) \dagger}\left(\gamma_{5}\right)_{\xi^{\prime} \alpha^{\prime}} \psi_{x b \beta}^{(k)} \psi_{y b^{\prime} \beta^{\prime}}^{(k) \dagger} \psi_{x c \gamma}^{(l)} \psi_{y c^{\prime} \gamma^{\prime}}^{(l) \dagger}\right. \\
& \left.\left.-\left(\gamma_{5}\right)_{\alpha \xi} \psi_{x a \xi}^{(n)} \psi_{y c^{\prime} \xi^{\prime}}^{(n) \dagger}\left(\gamma_{5}\right)_{\xi^{\prime} \gamma^{\prime}} \psi_{x b \beta}^{(k)} \psi_{y b^{\prime} \beta^{\prime}}^{(k) \dagger} \psi_{x c \gamma}^{(l)} \psi_{y a^{\prime} \alpha^{\prime}}^{(l) \dagger}\right]\right] .
\end{aligned}
$$

Using (22) the coefficients in front of the eigenfunction products can be written as 


$$
\begin{aligned}
& f_{n} f_{k} f_{l}+f_{-n} f_{-k} f_{-l}=2 \mathrm{i}\left(g_{n} g_{k} g_{l}-h_{n} h_{k} g_{h}-h_{n} g_{k} h_{l}-g_{n} h_{k} h_{l}\right), \\
& f_{n} f_{k} f_{-l}+f_{-n} f_{-k} f_{l}=2 \mathrm{i}\left(g_{n} g_{k} g_{l}-h_{n} h_{k} g_{h}+h_{n} g_{k} h_{l}+g_{n} h_{k} h_{l}\right), \\
& f_{n} f_{-k} f_{l}+f_{-n} f_{k} f_{-l}=2 \mathrm{i}\left(g_{n} g_{k} g_{l}+h_{n} h_{k} g_{h}-h_{n} g_{k} h_{l}+g_{n} h_{k} h_{l}\right), \\
& f_{-n} f_{k} f_{l}+f_{n} f_{-k} f_{-l}=2 \mathrm{i}\left(g_{n} g_{k} g_{l}+h_{n} h_{k} g_{h}+h_{n} g_{k} h_{l}-g_{n} h_{k} h_{l}\right) .
\end{aligned}
$$

Hence the difference of nucleon propagators with opposite parity contains no terms proportional to $h h h$ as indicated in Fig. 4.

$$
\text { APPENDIX C: } C\left(N_{ \pm}^{(1)}\right)-C\left(N_{ \pm}^{(2)}\right) \text { AND } C\left(N_{ \pm}^{(3)}\right)-C\left(N_{ \pm}^{(4)}\right)
$$

In order to prove that the propagators $C\left(N_{ \pm}^{(i)}\right)$ and $C\left(N_{ \pm}^{(i+1)}\right)$ for $i=1,3$ are also $g$ equivalent, we notice that from Table I we have $\Gamma_{2}^{(i+1)}=\gamma_{5} \Gamma_{2}^{(i)}$ and $\Gamma_{1}^{(i+1)}=\gamma_{5} \Gamma_{1}^{(i)}$. Therefore from Eq. (A2) and considering $i=1$, 3, we have

$$
\begin{aligned}
& C\left(N_{ \pm}^{(i)}\right)-C\left(N_{ \pm}^{(i+1)}\right) \\
& = \pm \epsilon_{a b c} \epsilon_{a^{\prime} b^{\prime} c^{\prime}}\left(\mathcal{P}_{ \pm}\right)_{\alpha^{\prime} \alpha}\left(\Gamma_{2}^{(i)}\right)_{\beta \gamma}\left(\Gamma_{2}^{(i)}\right)_{\gamma^{\prime} \beta^{\prime}} \sum_{n>0, k>0, l>0}\left[\left(f_{n} f_{k} f_{l}+f_{n} f_{-k} f_{-l}-f_{-n} f_{k} f_{-l}-f_{-n} f_{-k} f_{l}\right)\right. \\
& \times\left[\psi_{x a \alpha}^{(n)} \psi_{y a^{\prime} \alpha^{\prime}}^{(n) \dagger} \psi_{x b \beta}^{(k)} \psi_{y b^{\prime} \beta^{\prime}}^{(k) \dagger} \psi_{x c \gamma}^{(l)} \psi_{y c^{\prime} \gamma^{\prime}}^{(l) \dagger}-\left(\gamma_{5}\right)_{\alpha \xi} \psi_{x a \xi}^{(n)} \psi \psi_{y a^{\prime} \xi^{\prime}}^{(n) \dagger}\left(\gamma_{5}\right)_{\xi^{\prime} \alpha^{\prime}} \psi_{x b \beta}^{(k)} \psi_{y b^{\prime} \beta^{\prime}}^{(k) \dagger}\left(\gamma_{5}\right)_{\gamma \theta} \psi_{x c \theta}^{(l)} \psi_{y c^{\prime} \theta^{\prime}}^{(l) \dagger}\left(\gamma_{5}\right)_{\theta^{\prime} \gamma^{\prime}}\right] \\
& +\left(f_{n} f_{k} f_{-l}+f_{n} f_{-k} f_{l}-f_{-n} f_{k} f_{l}-f_{-n} f_{-k} f_{-l}\right) \times\left[\psi_{x a \alpha}^{(n)} \psi_{y a^{\prime} \alpha^{\prime}}^{(n) \dagger} \psi_{x b \beta}^{(k)} \psi_{y b^{\prime} \beta^{\prime}}^{(k) \dagger}\left(\gamma_{5}\right)_{\gamma \theta} \psi_{x c \theta}^{(l)} \psi_{y c^{\prime} \theta^{\prime}}^{(l) \dagger}\left(\gamma_{5}\right)_{\theta^{\prime} \gamma^{\prime}}\right. \\
& \left.-\left(\gamma_{5}\right)_{\alpha \xi} \psi_{x a \xi}^{(n)} \psi_{y a^{\prime} \xi^{\prime}}^{(n) \dagger}\left(\gamma_{5}\right)_{\xi^{\prime} \alpha^{\prime}} \psi_{x b \beta}^{(k)} \psi_{y b^{\prime} \beta^{\prime}}^{(k) \dagger} \psi_{x c \gamma}^{(l)} \psi_{y c^{\prime} \gamma^{\prime}}^{(l) \dagger}\right] \\
& -\left(f_{n} f_{k} f_{l}-f_{-n} f_{k} f_{-l}\right)\left[\psi_{x a \alpha}^{(n)} \psi_{y c^{\prime} \gamma^{\prime}}^{(n) \dagger} \psi_{x b \beta}^{(k)} \psi_{y b^{\prime} \beta^{\prime}}^{(k) \dagger} \psi_{x c \gamma}^{(l)} \psi_{y a^{\prime} \alpha^{\prime}}^{(l) \dagger}-\left(\gamma_{5}\right)_{\alpha \xi} \psi \psi_{x a \xi}^{(n)} \psi_{y c^{\prime} \xi^{\prime}}^{(n) \dagger}\left(\gamma_{5}\right)_{\xi^{\prime} \gamma^{\prime}} \psi_{x b \beta}^{(k)} \psi_{y b^{\prime} \beta^{\prime}}^{(k) \dagger}\left(\gamma_{5}\right)_{\gamma \theta} \psi_{x c \theta}^{(l)} \psi_{y a^{\prime} \theta^{\prime}}^{(l) \dagger}\left(\gamma_{5}\right)_{\theta^{\prime} \alpha^{\prime}}\right] \\
& -\left(f_{n} f_{k} f_{-l}-f_{-n} f_{k} f_{l}\right) \times\left[\psi_{x a \alpha}^{(n)} \psi_{y c^{\prime} \gamma^{\prime}}^{(n) \dagger} \psi_{x b \beta}^{(k)} \psi_{y b^{\prime} \beta^{\prime}}^{(k) \dagger}\left(\gamma_{5}\right)_{\gamma \theta} \psi_{x c \theta}^{(l)} \psi_{y a^{\prime} \theta^{\prime}}^{(l) \dagger}\left(\gamma_{5}\right)_{\theta^{\prime} \alpha^{\prime}}-\left(\gamma_{5}\right)_{\alpha \xi} \psi_{x a \xi}^{(n)} \psi_{y c^{\prime} \xi^{\prime}}^{(n) \dagger}\left(\gamma_{5}\right)_{\xi^{\prime} \gamma^{\prime}} \psi_{x b \beta}^{(k)} \psi_{y b^{\prime} \beta^{\prime}}^{(k) \dagger} \psi_{x c \gamma}^{(l)} \psi_{y a^{\prime} \alpha^{\prime}}^{(l) \dagger}\right] \\
& -\left(f_{n} f_{-k} f_{l}-f_{-n} f_{-k} f_{-l}\right) \times\left[\psi_{x a \alpha}^{(n)} \psi_{y c^{\prime} \gamma^{\prime}}^{(n) \dagger}\left(\gamma_{5}\right)_{\beta \omega} \psi_{x b \omega}^{(k)} \psi_{y b^{\prime} \omega^{\prime}}^{(k) \dagger}\left(\gamma_{5}\right)_{\omega^{\prime} \beta^{\prime}} \psi_{x c \gamma}^{(l)} \psi_{y a^{\prime} \alpha^{\prime}}^{(l) \dagger}\right. \\
& \left.-\left(\gamma_{5}\right)_{\alpha \xi} \psi_{x a \xi}^{(n)} \psi_{y c^{\prime} \xi^{\prime}}^{(n) \dagger}\left(\gamma_{5}\right)_{\xi^{\prime} \gamma^{\prime}}\left(\gamma_{5}\right)_{\beta \omega} \psi_{x b \omega}^{(k)} \psi_{y b^{\prime} \omega^{\prime}}^{(k) \dagger}\left(\gamma_{5}\right)_{\omega^{\prime} \beta^{\prime}}\left(\gamma_{5}\right)_{\gamma \theta} \psi_{x c \theta}^{(l)} \psi_{y a^{\prime} \theta^{\prime}}^{(l) \dagger}\left(\gamma_{5}\right)_{\theta^{\prime} \alpha^{\prime}}\right] \\
& -\left(f_{n} f_{-k} f_{-l}-f_{-n} f_{-k} f_{l}\right) \times\left[\psi_{x a \alpha}^{(n)} \psi_{y c^{\prime} \gamma^{\prime}}^{(n) \dagger}\left(\gamma_{5}\right)_{\beta \omega} \psi_{x b \omega}^{(k)} \psi_{y b^{\prime} \omega^{\prime}}^{(k) \dagger}\left(\gamma_{5}\right)_{\omega^{\prime} \beta^{\prime}}\left(\gamma_{5}\right)_{\gamma \theta} \psi_{x c \theta}^{(l)} \psi_{y a^{\prime} \theta^{\prime}}^{(l) \dagger}\left(\gamma_{5}\right)_{\theta^{\prime} \alpha^{\prime}}\right. \\
& \left.\left.-\left(\gamma_{5}\right)_{\alpha \xi} \psi_{x a \xi}^{(n)} \psi_{y c^{\prime} \xi^{\prime}}^{(n) \dagger}\left(\gamma_{5}\right)_{\xi^{\prime} \gamma^{\prime}}\left(\gamma_{5}\right)_{\beta \omega} \psi_{x b \omega}^{(k)} \psi_{y b^{\prime} \omega^{\prime}}^{(k) \dagger}\left(\gamma_{5}\right)_{\omega^{\prime} \beta^{\prime}} \psi_{x c \gamma}^{(l)} \psi_{y a^{\prime} \alpha^{\prime}}^{(l) \dagger}\right]\right]
\end{aligned}
$$

Using (22) we can rewrite the coefficients in front of the eigenfunction products in terms of $h_{n}$ and $g_{n}$, namely

$$
\begin{aligned}
f_{n} f_{k} f_{l}-f_{-n} f_{k} f_{-l} & =-2\left(h_{n} g_{k} g_{l}+\mathrm{i} h_{n} h_{k} g_{l}+g_{n} g_{k} h_{l}+\mathrm{i} g_{n} h_{k} h_{l}\right), \\
f_{n} f_{k} f_{-l}-f_{-n} f_{k} f_{l} & =-2\left(h_{n} g_{k} g_{l}+\mathrm{i} h_{n} h_{k} g_{l}-g_{n} g_{k} h_{l}-\mathrm{i} g_{n} h_{k} h_{l}\right), \\
f_{n} f_{-k} f_{l}-f_{-n} f_{-k} f_{-l} & =-2\left(h_{n} g_{k} g_{l}-\mathrm{i} h_{n} h_{k} g_{l}+g_{n} g_{k} h_{l}-\mathrm{i} g_{n} h_{k} h_{l}\right), \\
f_{n} f_{-k} f_{-l}-f_{-n} f_{-k} f_{l} & =-2\left(h_{n} g_{k} g_{l}-\mathrm{i} h_{n} h_{k} g_{l}-g_{n} g_{k} h_{l}+\mathrm{i} g_{n} h_{k} h_{l}\right) .
\end{aligned}
$$

Therefore as we can see from Eqs. (C1) and (C2) the differences $C\left(N_{ \pm}^{(1)}\right)-C\left(N_{ \pm}^{(2)}\right)$ and $C\left(N_{ \pm}^{(3)}\right)-C\left(N_{ \pm}^{(4)}\right)$ are only proportional to $g g h$ and $g h h$ terms, as indicated in Fig. 4. 
[1] C. B. Lang and M. Schrock, Phys. Rev. D 84, 087704 (2011).

[2] L. Y. Glozman, C. B. Lang, and M. Schrock, Phys. Rev. D 86, 014507 (2012).

[3] M. Denissenya, L. Y. Glozman, and C. B. Lang, Phys. Rev. D 89, 077502 (2014).

[4] M. Denissenya, L. Y. Glozman, and C. B. Lang, Phys. Rev. D 91, 034505 (2015).

[5] M. Denissenya, L. Y. Glozman, and M. Pak, Phys. Rev. D 91, 114512 (2015).

[6] M. Denissenya, L. Y. Glozman, and M. Pak, Phys. Rev. D 92, 074508 (2015); 92, 099902(E) (2015).

[7] L. Y. Glozman, Eur. Phys. J. A 51, 27 (2015).

[8] L. Y. Glozman and M. Pak, Phys. Rev. D 92, 016001 (2015).

[9] C. Rohrhofer, Y. Aoki, G. Cossu, H. Fukaya, L. Y. Glozman, S. Hashimoto, C. B. Lang, and S. Prelovsek, Phys. Rev. D 96, 094501 (2017); 99, 039901(E) (2019).
[10] C. Rohrhofer, Y. Aoki, G. Cossu, H. Fukaya, C. Gattringer, L. Y. Glozman, S. Hashimoto, C. B. Lang, and S. Prelovsek, arXiv:1902.03191.

[11] L. Y. Glozman, Eur. Phys. J. A 54, 117 (2018).

[12] A. Tomiya, G. Cossu, S. Aoki, H. Fukaya, S. Hashimoto, T. Kaneko, and J. Noaki, Phys. Rev. D 96, 034509 (2017); 96, 079902(A) (2017).

[13] T. Banks and A. Casher, Nucl. Phys. B169, 103 (1980).

[14] T. D. Cohen, Phys. Rev. D 54, R1867 (1996).

[15] C. B. Lang, Phys. Rev. D 97, 114510 (2018).

[16] M. Catillo and L. Y. Glozman, Phys. Rev. D 98, 014030 (2018).

[17] K. Nagata, A. Hosaka, and V. Dmitrasinovic, Eur. Phys. J. C 57, 557 (2008). 